\title{
The inverse problem for some special spectral data
}

\author{
V. O. Vakhnenko ${ }^{a, *}$, E. J. Parkes ${ }^{b}$ \\ ${ }^{a}$ Institute of Geophysics, National Academy of Sciences of Ukraine, 01054 Kÿ̈v, Ukraine \\ ${ }^{b}$ Department of Mathematics 83 Statistics, University of Strathclyde, Glasgow G1 1XH, \\ $U K$
}

\begin{abstract}
In this paper the spectral problem of third order for the inverse scattering transform (IST) method is solved. For the discrete part of the spectral data, the two-multiple poles are taken into account. The line spectrum of continuum states for the IST method is examined as well. The suggested spectrum approximates in first order the step-function. The scope for the suggested spectral data is demonstrated through the analysis of the Vakhnenko-Parkes equation that allows new solutions to be obtained. The account of the timedependence is different from the standard procedure.
\end{abstract}

Keywords: inverse problem, spectral data, poles of second order, line spectrum of continuum states

PACS: 00.30.Lk, 02.30.Jr, 05.45.Yv

\section{Introduction}

The inverse scattering transform (IST) method is one of the fundamental methods for solving various nonlinear evolution equations. The method enables one to solve the initial value problem for a nonlinear evolution equation. Moreover, it provides a proof of the complete integrability of the equation. The essence of the application of the IST is as follows. The equation of interest for study is written as the compatibility condition for two linear equations (the Lax pair). Then the initial condition is mapped into the scattering data. It is important that the spectrum always retains constant values. The time

\footnotetext{
*Corresponding author

Email addresses: vakhnenko@ukr.net (V. O. Vakhnenko), e.j.parkes@strath.ac.uk (E. J. Parkes)
} 
evolution of scattering data is simple and linear. From a knowledge of scattering data evolution, the solution is reconstructed. Hence, for this method the direct spectral problem and the inverse spectral problem are considered. The latter consists of reconstructing the solution of the nonlinear equation from the spectral data $(\S 2)$. In the general case it is necessary to analyze both the discrete part and the continuum part of the spectral data. It is well-known that the discrete part is associated with soliton solutions, while the continuum part of the spectral data is related to the periodical solutions. For the spectrum of bound states, we take into account the two-multiple poles ( $(3)$, while for continuum states, a special form of the spectral data is considered $(\S 4)$. The spectrum of continuum states is taken as a line spectrum that in first order approximates the step-function $(\S 4)$. The problem of reconstructing the solution from the spectral data is considered in $\S 5$ and $\S 6$. The solution for discrete spectral data with two-multiple poles is taken in $\S 7$.

\section{The spectral problem}

The inverse problem for $N \times N$ spectral equations has been considered by Caudrey [1-3] and Kaup [4]. Following the method described by Caudrey [1], the spectral equation for many evolution equations can be written

$$
\frac{\partial}{\partial X} \boldsymbol{\psi}=[\mathbf{A}(\zeta)+\mathbf{B}(X, \zeta)] \cdot \boldsymbol{\psi}
$$

For the sake of convenience, we study the third-order form of the spectral equation (2.1). The third-order spectral equation is associated with a Boussinesq equation [1-6], a higher order $\mathrm{KdV}$ equation [4, 7], a model equation for shallow water waves [8, 9], and the Vakhnenko-Parkes equation (VPE) [10-14]. The VPE arises from another nonlinear integrable equation, named the Vakhnenko equation (VE) $[15,16]$

$$
\frac{\partial}{\partial x}\left(\frac{\partial}{\partial t}+u \frac{\partial}{\partial x}\right) u+u=0
$$

after an appropriate change of variables [10, 11, 17, 18].

It is interesting to note that equation (2.2) follows as a particular limit of the following generalized Korteweg-de Vries equation

$$
\frac{\partial}{\partial x}\left(\frac{\partial u}{\partial t}+u \frac{\partial u}{\partial x}-\beta \frac{\partial^{3} u}{\partial x^{3}}\right)=\gamma u
$$


derived by Ostrovsky [19] to model small-amplitude long waves in a rotating fluid ( $\gamma u$ is induced by the Coriolis force) of finite depth. Subsequently, equation (2.2) was known by different names in the literature, such as the Ostrovsky-Hunter equation, the short-wave equation, the reduced Ostrovsky equation and the Ostrovsky-Vakhnenko equation depending on the physical context in which it is studied.

Using the VPE [10-14]

$$
W_{X X T}+\left(1+W_{T}\right) W_{X}=0
$$

or, in equivalent form with $U \equiv W_{X}$,

$$
U U_{X X T}-U_{X} U_{X T}+U^{2} U_{T}=0
$$

as an example, we aim to examine both the two-multiple poles and some special forms of the spectral data for which the inverse problem can be solved.

After the Lax pair

$$
\begin{aligned}
& \psi_{X X X}+W_{X} \psi_{X}-\lambda \psi=0, \\
& 3 \psi_{X T}+\left(1+W_{T}\right) \psi+\mu \psi_{X}=0
\end{aligned}
$$

for the VPE was derived in [10], in [20] the Lax pair was written in its original variables as a zero curvature condition. Moreover, in [20] Hone and Wang have shown that there is a subtle connection between the Sawada-Kotera hierarchy and the VE, between the Degasperis-Procesi equation (DPE) and the VE (see also [21]), and between the Lax pairs of the DPE and the VE.

As expected, (2.4) and (2.5) are similar to, but cannot be transformed into, the corresponding equations for the Hirota-Satsuma equation (HSE) (see Eqs. (A8a) and (A8b) in [22]). Clarkson and Mansfield [23] note that the scattering problem for the HSE is similar to that for the Boussinesq equation which has been studied comprehensively by Deift et al. [6].

The explicit soliton solution for the VPE was obtained by the IST method in [10] and by the Hirota method in [17, 18, 24] whereas, for the Cauchy problem at long-time, the IST approach was presented for a Riemann-Hilbert problem $[25,26]$ in original (physical) independent variables for the $\mathrm{VE}$ in [26]. As usual, the transformation between the solution of the VPE and the $\mathrm{VE}$ is in the form (2.12), (2.13) in [17].

The spectral equation (2.4) has a matrix form (2.1) with

$$
\boldsymbol{\psi}=\left(\begin{array}{c}
\psi \\
\psi_{X} \\
\psi_{X X}
\end{array}\right), \quad \mathbf{A}=\left(\begin{array}{ccc}
0 & 1 & 0 \\
0 & 0 & 1 \\
\lambda & 0 & 0
\end{array}\right), \quad \mathbf{B}=\left(\begin{array}{ccc}
0 & 0 & 0 \\
0 & 0 & 0 \\
0 & -W_{X} & 0
\end{array}\right)
$$


The matrix $\mathbf{A}$ has eigenvalues $\lambda_{j}(\zeta)$ and left- and right-eigenvectors $\tilde{\boldsymbol{v}}_{j}(\zeta)$ and $\boldsymbol{v}_{j}(\zeta)$, respectively. These quantities are defined through a spectral parameter $\lambda$ as

$$
\begin{aligned}
& \lambda_{j}(\zeta)=\omega_{j} \zeta, \quad \lambda_{j}^{3}(\zeta)=\lambda \\
& \boldsymbol{v}_{j}(\zeta)=\left(\begin{array}{c}
1 \\
\lambda_{j}(\zeta) \\
\lambda_{j}^{2}(\zeta)
\end{array}\right), \quad \tilde{\boldsymbol{v}}_{j}(\zeta)=\left(\begin{array}{lll}
\lambda_{j}^{2}(\zeta) & \lambda_{j}(\zeta) & 1
\end{array}\right),
\end{aligned}
$$

where $\omega_{j}=e^{2 \pi \mathrm{i}(j-1) / 3}$ are the cube roots of $1(j=1,2,3)$. Obviously the $\lambda_{j}(\zeta)$ are distinct and they and $\tilde{\boldsymbol{v}}_{j}(\zeta)$ and $\boldsymbol{v}_{j}(\zeta)$ are analytic throughout the complex $\zeta$-plane.

The solution of the system of the linear equations (2.1) has been obtained by Caudrey $[1,3]$ in terms of Jost functions $\phi_{j}(X, \zeta)$ which have the asymptotic behaviour

$$
\boldsymbol{\Phi}_{j}(X, \zeta):=\exp \left\{-\lambda_{j}(\zeta) X\right\} \boldsymbol{\phi}_{j}(X, \zeta) \rightarrow \boldsymbol{v}_{j}(\zeta) \text { as } X \rightarrow-\infty .
$$

Caudrey [1] showed how the Eq. (2.1) can be solved by expressing it as a Fredholm integral equation.

The complex $\zeta$-plane is to be divided into regions such that, in the interior of each region, the order of the numbers $\operatorname{Re}\left(\lambda_{i}(\zeta)\right)$ is fixed (see Fig. 1). As we pass from one region to another this order changes and hence, on a boundary between two regions, $\operatorname{Re}\left(\lambda_{i}(\zeta)\right)=\operatorname{Re}\left(\lambda_{j}(\zeta)\right)$ for at least one pair $i \neq j$. The Jost function $\phi_{j}$ is regular throughout the complex $\zeta$-plane apart from poles and finite singularities on the boundaries between the regions. At any point in the interior of any region of the complex $\zeta$-plane, the solution of Eq. (2.1) is obtained by the relation (2.12) from [1]. It is the direct spectral problem.

However, we will start from preassigned spectral data for reconstructing the solution $W$ of the analyzed nonlinear equation, i.e. we will consider only the inverse spectral problem. The information about the singularities of the Jost functions $\phi_{j}(X, \zeta)$ reside in the spectral data. First let us consider the poles. It is assumed that a pole $\zeta_{i}^{(k)}$ of $\phi_{i}(X, \zeta)$ does not coincide with a pole of $\phi_{j}(X, \zeta), j \neq i$ and does not lie on a boundary between two regions. Note that, for $\phi_{j}\left(X, \zeta_{i}^{(k)}\right)$, the point $\zeta_{i}^{(k)}$ lies in the interior of a regular region. We will need the well-known relations for simple poles [1,3] in order to compare them with new results which will be obtained in Sec.3. As proven in [1], the 
residue of a simple pole can be calculated as

$$
\operatorname{Res} \phi_{i}\left(X, \zeta_{i}^{(k)}\right)=\sum_{\substack{j=1 \\ j \neq i}}^{n} \gamma_{i j}^{(k)} \phi_{j}\left(X, \zeta_{i}^{(k)}\right)
$$

The quantities $\zeta_{i}^{(k)}$ and $\gamma_{i j}^{(k)}$ constitute the discrete part of the spectral data in the case of simple poles.

In contrast to the papers $[1,3]$ we do not restrict ourselves to simple poles. Indeed, one of the results we will prove in the next section is that the two-multiple poles can be taken into account in the discrete part of the spectral data.

Now we consider the singularities on the boundaries between regions. However, in order to simplify matters, we first make some observations. The solution of the spectral problem can be facilitated by using various symmetry properties. In view of (2.1), we need only consider the first elements of

$$
\phi_{i}(X, \zeta)=\left(\begin{array}{c}
\phi_{i}(X, \zeta) \\
\phi_{i}(X, \zeta)_{X} \\
\phi_{i}(X, \zeta)_{X X}
\end{array}\right)
$$

while the symmetry

$$
\phi_{1}\left(X, \zeta / \omega_{1}\right)=\phi_{2}\left(X, \zeta / \omega_{2}\right)=\phi_{3}\left(X, \zeta / \omega_{3}\right)
$$

means we need only consider $\phi_{1}(X, \zeta)$. In our case, for $\phi_{1}(X, \zeta)$, the complex $\zeta$-plane is divided into four regions by two lines (see Fig. 1 ) given by

(i) $\zeta^{\prime}=\omega_{2} \xi$, where $\operatorname{Re}\left(\lambda_{1}(\zeta)\right)=\operatorname{Re}\left(\lambda_{2}(\zeta)\right)$,

(ii) $\zeta^{\prime}=-\omega_{3} \xi$, where $\operatorname{Re}\left(\lambda_{1}(\zeta)\right)=\operatorname{Re}\left(\lambda_{3}(\zeta)\right)$,

where $\xi$ is real. The singularity of $\phi_{1}(X, \zeta)$ can appear only on these boundaries between the regular regions on the $\zeta$-plane and it is characterized by functions $Q_{1 j}\left(\zeta^{\prime}\right)$ at each fixed $j \neq 1$. We denote the limit of a quantity, as the boundary is approached, by the superfix \pm in according to the sign of $\operatorname{Re}\left(\lambda_{1}(\zeta)-\lambda_{j}(\zeta)\right)$ (see Fig. 1).

In [1] (see Eq. (3.14) there) the jump of $\phi_{1}(X, \zeta)$ on the boundaries is calculated as

$$
\phi_{1}^{+}(X, \zeta)-\phi_{1}^{-}(X, \zeta)=\sum_{j=2}^{3} Q_{1 j}(\zeta) \phi_{j}^{-}(X, \zeta),
$$


where, from (2.12), the sum is over the lines $\zeta^{\prime}=\omega_{2} \xi$ and $\zeta^{\prime}=-\omega_{3} \xi$ given by

(i) $\quad \zeta^{\prime}=\omega_{2} \xi, \quad$ with $\quad Q_{12}^{(1)}\left(\zeta^{\prime}\right) \neq 0, \quad Q_{13}^{(1)}\left(\zeta^{\prime}\right) \equiv 0$,

(ii) $\zeta^{\prime}=-\omega_{3} \xi, \quad$ with $\quad Q_{12}^{(2)}\left(\zeta^{\prime}\right) \equiv 0, \quad Q_{13}^{(2)}\left(\zeta^{\prime}\right) \neq 0$.

The quantities $Q_{1 j}\left(\zeta^{\prime}\right)$ along all the boundaries constitute the continuum part of the spectral data.

Thus, for simple poles, the spectral data are $[1,3]$

$$
S=\left\{\zeta_{1}^{(k)}, \gamma_{1 j}^{(k)}, Q_{1 j}\left(\zeta^{\prime}\right) ; j=2,3, k=1,2, \ldots, m\right\} .
$$

One of the important features which is to be noted for the IST method is as follows. After the spectral data have been obtained, we need to seek the time-evolution of the spectral data. In Refs. [10-14] it is proved that for the VPE the $T$-dependence is revealed as

$$
\phi_{i}(X, T, \zeta)=\exp \left[-\left(3 \lambda_{i}(\zeta)\right)^{-1} T\right] \phi_{i}(X, 0, \zeta),
$$

then for spectral data (2.15)

$$
\begin{aligned}
& \zeta_{j}^{(k)}(T)=\zeta_{j}^{(k)}(0), \\
& \gamma_{1 j}^{(k)}(T)=\gamma_{1 j}^{(k)}(0) \exp \left\{\left[-\left(3 \lambda_{j}\left(\zeta_{1}^{(k)}\right)\right)^{-1}+\left(3 \lambda_{1}\left(\zeta_{1}^{(k)}\right)\right)^{-1}\right] T\right\}, \\
& Q_{1 j}\left(T ; \zeta^{\prime}\right)=Q_{1 j}\left(0 ; \zeta^{\prime}\right) \exp \left\{\left[-\left(3 \lambda_{j}\left(\zeta^{\prime}\right)\right)^{-1}+\left(3 \lambda_{1}\left(\zeta^{\prime}\right)\right)^{-1}\right] T\right\} .
\end{aligned}
$$

The final step in the application of the IST method is to reconstruct the matrix $B(X, T ; \zeta)$ and the solution $W(X, T)$ from the spectral data $S(2.15)$.

Caudrey has proved that for simple poles the spectral data define $\Phi_{1}(X, \zeta)$ uniquely in the form (see Eq. (6.20) in [1]))

$$
\Phi_{1}(X, T ; \zeta)=1-\Omega_{d}(X, T ; \zeta)+\Omega_{c}(X, T ; \zeta),
$$

where

$$
\begin{aligned}
\Omega_{d}(X, T ; \zeta) \equiv & \sum_{k=1}^{K} \sum_{j=2}^{3} \gamma_{1 j}^{(k)}(T) \frac{\exp \left\{\left[\lambda_{j}\left(\zeta_{1}^{(k)}\right)-\lambda_{1}\left(\zeta_{1}^{(k)}\right)\right] X\right\}}{\lambda_{1}\left(\zeta_{1}^{(k)}\right)-\lambda_{1}(\zeta)} \\
& \times \Phi_{1}\left(X, T ; \omega_{j} \zeta_{1}^{(k)}\right),
\end{aligned}
$$




$$
\begin{aligned}
\Omega_{c}(X, T ; \zeta) \equiv & \frac{1}{2 \pi \mathrm{i}} \int \sum_{j=2}^{3} Q_{1 j}\left(T ; \zeta^{\prime}\right) \frac{\exp \left\{\left[\lambda_{j}\left(\zeta^{\prime}\right)-\lambda_{1}\left(\zeta^{\prime}\right)\right] X\right\}}{\zeta^{\prime}-\zeta} \\
& \times \Phi_{1}^{-}\left(X, T ; \omega_{j} \zeta^{\prime}\right) d \zeta^{\prime}
\end{aligned}
$$

Equations (2.17)-(2.19) contains the spectral data, namely $K$ simple poles with the quantities $\gamma_{1 j}^{(k)}$ for the bound state spectrum as well as the functions $Q_{1 j}\left(\zeta^{\prime}\right)$ given along all the boundaries of regular regions for the continuous spectrum. The integral in (2.18) is along all the boundaries (see the dashed lines in Fig. 1). The direction of integration is taken so that the side chosen to be $\operatorname{Re}\left(\lambda_{1}(\zeta)-\lambda_{j}(\zeta)\right)<0$ is shown by the arrows in Fig. 1 (for the lines (2.12), $\xi$ sweeps from $-\infty$ to $+\infty)$.

It is necessary to note that we should carry out the integration along the lines $\omega_{2}(\xi+\mathrm{i} \varepsilon)$ and $-\omega_{3}(\xi+\mathrm{i} \varepsilon)$ with $\varepsilon>0$. In this case the condition (2.8) is satisfied. Passing to the limit $\varepsilon \rightarrow 0$ we can obtain the periodical solution which does not satisfy the condition (2.8). However, for any finite $\varepsilon>0$, the restricted region on $X$ can be determined where the solution associated with a finite $\varepsilon>0$ (for which the condition (2.8) is valid) and the solution associated with $\varepsilon=0$ are sufficiently close to each other. In this sense, taking the integration at $\varepsilon=0$, we remain within the inverse scattering theory [1], and so the condition (2.8) can be omitted. The solution obtained at $\varepsilon=0$ can be extended to sufficiently large finite $X$. Thus, we will interpret the solution obtained at $\varepsilon=0$ as the solution of the VPE (2.3) which is valid for arbitrary but finite $X$.

With appropriate choice of values for $\zeta$, the left-hand side in $(2.17)$ can be $\Phi_{1}\left(X, T ; \omega_{j} \zeta_{1}^{(k)}\right)$, or by allowing $\zeta$ to approach the boundaries from the appropriate sides, the left-hand side can be $\Phi_{1}^{-}\left(X, T ; \omega_{j} \zeta^{\prime}\right)$. We acquire a set of linear matrix/Fredholm equations in the unknowns $\Phi_{1}\left(X, T ; \omega_{j} \zeta_{1}^{(k)}\right)$ and $\Phi_{1}^{-}\left(X, T ; \omega_{j} \zeta^{\prime}\right)[1]$. The solution of this equation system enables one to define $\Phi_{1}(X, T ; \zeta)$ from $(2.17)$.

By knowing $\Phi_{1}(X, T ; \zeta)$, we can take extra information into account, namely that the expansion of $\Phi_{1}(X, T ; \zeta)$ as an asymptotic series in $\lambda_{1}^{-1}(\zeta)$ connects with $W(X, T)$ as follows (cf. Eq. (2.7) in [4]):

$$
\Phi_{1}(X, T ; \zeta)=1-\frac{1}{3 \lambda_{1}(\zeta)}[W(X, T)-W(-\infty)]+O\left(\lambda_{1}^{-2}(\zeta)\right) .
$$

Consequently, the solution $W(X, T)$ and the matrix $B(X, T ; \zeta)$ can be reconstructed from the spectral data. 
In the remaining sections we will study both the multiple poles for the discrete part of spectral data and the continuum part of the spectral data in special form. Apart from the relation (2.18), all other formulas are true for the suggested spectral data and will be used subsequently.

\section{The two-multiple poles}

For single poles the formula (2.18) are true. Now we take into account the two-multiple poles. Let us consider the additional equation to the spectral equation (2.4)

$$
\chi_{X X X}+W_{\zeta X} \psi_{X}+W_{X} \chi_{X}-\zeta^{3} \chi-3 \zeta^{2} \psi=0 .
$$

For $\chi=\psi_{\zeta}$ the equation (3.1) stems from (2.4) by differentiation with respect to $\zeta$. For convenience, the spectral parameter $\lambda$ is written as $\lambda=\zeta^{3}$ by virtue of (2.7).

The matrix form of the system of equations (2.4) and (3.1) is as (2.1) with

$$
\begin{aligned}
\boldsymbol{\psi} & =\left(\begin{array}{c}
\psi \\
\psi_{X} \\
\psi_{X X} \\
\chi \\
\chi_{X} \\
\chi_{X X}
\end{array}\right), \quad \mathbf{A}=\left(\begin{array}{ccccccc}
0 & 1 & 0 & 0 & 0 & 0 \\
0 & 0 & 1 & 0 & 0 & 0 \\
\zeta^{3} & 0 & 0 & 0 & 0 & 0 \\
0 & 0 & 0 & 0 & 1 & 0 \\
0 & 0 & 0 & 0 & 0 & 1 \\
3 \zeta^{2} & 0 & 0 & \zeta^{3} & 0 & 0
\end{array}\right) \\
\mathbf{B} & =\left(\begin{array}{cccccc}
0 & 0 & 0 & 0 & 0 & 0 \\
0 & 0 & 0 & 0 & 0 & 0 \\
0 & -W_{X} & 0 & 0 & 0 & 0 \\
0 & 0 & 0 & 0 & 0 & 0 \\
0 & 0 & 0 & 0 & 0 & 0 \\
0 & -W_{\zeta X} & 0 & 0 & -W_{X} & 0
\end{array}\right) .
\end{aligned}
$$

The matrix $\mathbf{A}$ has three pairs of 2-multiple eigenvalues and right-eigenvectors

$$
\begin{gathered}
\lambda_{j}(\zeta)=\lambda_{j+3}(\zeta), \quad \lambda_{j}(\zeta)=\omega_{j} \zeta, \quad \lambda_{j}^{3}(\zeta)=\lambda, \\
\boldsymbol{v}_{j}(\zeta)=\left(\begin{array}{c}
0 \\
0 \\
0 \\
1 \\
\lambda_{j}(\zeta) \\
\lambda_{j}^{2}(\zeta)
\end{array}\right)=\boldsymbol{v}_{j+3}(\zeta)=\left(\begin{array}{c}
0 \\
0 \\
0 \\
1 \\
\lambda_{j+3}(\zeta) \\
\lambda_{j+3}^{2}(\zeta)
\end{array}\right), \quad j=1,2,3 .
\end{gathered}
$$


It is known [27] that for system $(2.4),(3.1)$ at $W=0$ to every pair of vectors $\boldsymbol{v}_{j}(\zeta), \boldsymbol{v}_{j+3}(\zeta)(j=1,2,3)$, there corresponds a system of solutions

$$
\boldsymbol{\psi}_{j}=\boldsymbol{v}_{j} \exp \left(\lambda_{j} X\right), \quad \boldsymbol{\psi}_{j+3}=\left(\boldsymbol{v}_{j}+X \boldsymbol{v}_{2 j}\right) \exp \left(\lambda_{j} X\right),
$$

where (see p. 97 in [27])

$$
\mathbf{A} \boldsymbol{v}_{j}=\lambda_{j} \boldsymbol{v}_{j}, \quad \mathbf{A} \boldsymbol{v}_{2 j}=\lambda_{j} \boldsymbol{v}_{2 j}+\boldsymbol{v}_{j} .
$$

The multiplicity of eigenvalues does not allow us to obtain the fundamental system of solutions for the system (2.4), (3.1). To avoid this obstacle we introduce the equation

$$
\chi_{X X X}+W_{\zeta X} \psi_{X}+W_{X} \chi_{X}-(\zeta+\varepsilon)^{3} \chi-3(\zeta+\varepsilon)^{2} \psi=0
$$

instead of equation (3.1). The system (2.4), (3.6) in matrix form has the matrix

$$
\mathbf{A}=\left(\begin{array}{cccccc}
0 & 1 & 0 & 0 & 0 & 0 \\
0 & 0 & 1 & 0 & 0 & 0 \\
\zeta^{3} & 0 & 0 & 0 & 0 & 0 \\
0 & 0 & 0 & 0 & 1 & 0 \\
0 & 0 & 0 & 0 & 0 & 1 \\
3(\zeta+\varepsilon)^{2} & 0 & 0 & (\zeta+\varepsilon)^{3} & 0 & 0
\end{array}\right)
$$

with different eigenvalues and right-eigenvectors, which in the first approximation $O(\varepsilon)$ have the forms

$$
\begin{aligned}
& \lambda_{j}(\zeta)=\omega_{j} \zeta, \quad \lambda_{j+3}(\zeta)=\omega_{j}(\zeta+\varepsilon), \\
& \boldsymbol{v}_{j}(\zeta)=\left(\begin{array}{c}
-\varepsilon \\
-\varepsilon \lambda_{j} \\
-\varepsilon \lambda_{j}^{2} \\
1 \\
\lambda_{j} \\
\lambda_{j}^{2}
\end{array}\right), \quad \boldsymbol{v}_{j+3}(\zeta)=\left(\begin{array}{c}
0 \\
0 \\
0 \\
1 \\
\lambda_{j+3}^{2} \\
\lambda_{j+3}^{2}
\end{array}\right), \quad j=1,2,3 .
\end{aligned}
$$

As $\varepsilon \rightarrow 0$ the relations (3.8) tend to (3.3). At $W=0$ the solutions of system $(2.4),(3.6)$ are

$$
\boldsymbol{\psi}_{j}=\boldsymbol{v}_{j} \exp \left(\lambda_{j} X\right), \quad j=1 \ldots 6 .
$$

In the accepted approximation $O(\varepsilon)$, we take $\boldsymbol{\psi}_{j+3}=\boldsymbol{v}_{j+3}\left(1+\omega_{j} \varepsilon X\right) \exp \left(\lambda_{j} X\right)$ (here $j=1,2,3)$ then (3.9) is in accord with (3.4). 
Since the eigenvalues (3.8) for matrix A (3.7) are different, we can state that a fundamental system of solutions for the system of the equations (2.4), (3.6) exists (here, for the sake of convenience, the variable $X$ is omitted), namely

$$
\phi_{j}\left(\lambda_{j}(\zeta)\right), \quad j=1 \ldots 6 .
$$

According to $[1,3]$ we consider the Wronskian

$$
W r=\operatorname{det}\left[\phi_{1}\left(\lambda_{1}\right), \phi_{2}\left(\lambda_{2}\right), \ldots, \phi_{6}\left(\lambda_{6}\right)\right] .
$$

If the Wronskian $W r$ is non-zero at least at one point $X_{0}$, then it is proved in [27] (see p. 132 there) to be finite and non-zero even when $\zeta$ approaches a pole.

Let $\phi_{1}\left(\lambda_{1}(\zeta)\right)$ have poles at $\zeta=\zeta_{1}^{(k)},(k=1,2)$. Then $\left(\zeta-\zeta_{1}^{(k)}\right) W r=$ $\operatorname{det}\left[\left(\zeta-\zeta_{1}^{(k)}\right) \phi_{1}\left(\lambda_{1}\right), \phi_{2}\left(\lambda_{2}\right), \ldots, \phi_{6}\left(\lambda_{6}\right)\right]$ and taking the limit $\zeta \rightarrow \zeta_{1}^{(k)}$ we obtain

$$
0=\operatorname{det}\left[\operatorname{Res} \phi_{1}\left(\lambda_{1}\right), \phi_{2}\left(\lambda_{2}\right), \ldots, \phi_{6}\left(\lambda_{6}\right)\right] .
$$

Thus the columns (vectors) are linearly dependent. The dependence on the vector $\phi_{4}\left(\lambda_{4}\right)$ is omitted, since it has the same poles as $\phi_{1}\left(\lambda_{1}\right)$ at $\varepsilon \rightarrow 0$.

As a result from (3.12), we obtain the solution of the spectral equation (2.4) for the bound state spectrum

$$
\begin{aligned}
\Phi_{1}(X ; \zeta)= & 1-\sum_{k=1}^{2} \sum_{j=2}^{3}\left[\tilde{\gamma}_{1 j}^{(k)} \frac{\exp \left\{\left[\lambda_{j}\left(\zeta_{1}^{(k)}\right)-\lambda_{1}\left(\zeta_{1}^{(k)}\right)\right] X\right\}}{\lambda_{1}\left(\zeta_{1}^{(k)}\right)-\lambda_{1}(\zeta)}\right. \\
& \times \Phi_{1}\left(X ; \omega_{j} \zeta_{1}^{(k)}\right) \\
& +\tilde{\gamma}_{1 j+3}^{(k)} \frac{\exp \left\{\left[\lambda_{j}\left(\zeta_{1}^{(k)}+\varepsilon^{(k)}\right)-\lambda_{1}\left(\zeta_{1}^{(k)}+\varepsilon^{(k)}\right)\right] X\right\}}{\lambda_{1}\left(\zeta_{1}^{(k)}+\varepsilon^{(k)}\right)-\lambda_{1}(\zeta)} \\
& \left.\times \Phi_{1}\left(X ; \omega_{j}\left(\zeta_{1}^{(k)}+\varepsilon^{(k)}\right)\right)\right]
\end{aligned}
$$


By expanding the functions depending on $\varepsilon^{(k)}$ in series within accuracy of $O\left(\varepsilon^{(k)}\right)$, we rewrite the solution

$$
\begin{aligned}
\Phi_{1}(X ; \zeta)= & 1-\sum_{k=1}^{2} \sum_{j=2}^{3}\left\{\gamma_{1 j}^{(k)} \frac{\exp \left\{\left[\lambda_{j}\left(\zeta_{1}^{(k)}\right)-\lambda_{1}\left(\zeta_{1}^{(k)}\right)\right] X\right\}}{\lambda_{1}\left(\zeta_{1}^{(k)}\right)-\lambda_{1}(\zeta)}\right. \\
& \times \Phi_{1}\left(X ; \omega_{j} \zeta_{1}^{(k)}\right) \\
& +\frac{\partial}{\partial \zeta_{1}^{(k)}}\left[\gamma_{1 j+3}^{(k)} \frac{\exp \left\{\left[\lambda_{j}\left(\zeta_{1}^{(k)}\right)-\lambda_{1}\left(\zeta_{1}^{(k)}\right)\right] X\right\}}{\lambda_{1}\left(\zeta_{1}^{(k)}\right)-\lambda_{1}(\zeta)}\right. \\
& \left.\left.\times \Phi_{1}\left(X ; \omega_{j} \zeta_{1}^{(k)}\right)\right]\right\},
\end{aligned}
$$

where $\gamma_{1 j}^{(k)}=\tilde{\gamma}_{1 j}^{(k)}+\tilde{\gamma}_{1 j+3}^{(k)}, \gamma_{1 j+3}^{(k)}=\varepsilon^{(k)} \tilde{\gamma}_{1 j+3}^{(k)}$. It is important to note that the solution (3.14) is independent of $\varepsilon^{(k)}$ now.

The relationship (3.14) formally passes into (5.1), (5.2) with appropriate change of variables. For this reason the reconstruction of the solution $W$ for (3.14) is similar to the problem we will consider for the special form of continuum states (5.1).

\section{Special form for the continuum part of the spectral data}

Now we consider the continuous spectrum of the associated eigenvalue problem (2.1), (2.6), (2.7), i.e. assume that at least some of the functions $Q_{1 j}\left(\zeta^{\prime}\right)$ are non-zero. At each fixed $j \neq 1$ the functions $Q_{1 j}\left(\zeta^{\prime}\right)$ characterize the singularity of $\Phi_{1}(X, \zeta)$. As we have shown, this singularity can appear only on boundaries between the regular regions on the $\zeta$-plane, where the condition $\operatorname{Re}\left(\lambda_{1}\left(\zeta^{\prime}\right)-\lambda_{j}\left(\zeta^{\prime}\right)\right)=0$ defines these boundaries [1]. For the VPE (2.3), as we know, the complex $\zeta$-plane is divided into four regions by two lines $(2.14)$

(i) $\zeta^{\prime}=\omega_{2} \xi, \quad$ with $\quad Q_{12}^{(1)}\left(\zeta^{\prime}\right) \neq 0, \quad Q_{13}^{(1)}\left(\zeta^{\prime}\right) \equiv 0$,

(ii) $\zeta^{\prime}=-\omega_{3} \xi, \quad$ with $\quad Q_{12}^{(2)}\left(\zeta^{\prime}\right) \equiv 0, \quad Q_{13}^{(2)}\left(\zeta^{\prime}\right) \neq 0$,

where $\xi$ is real (see Fig. 1) and sweeps from $-\infty$ to $+\infty$. 
Recently in [12-14] we have considered the singularity functions $Q_{1 j}\left(\zeta^{\prime}\right)$ on the boundaries, on which the Jost function $\phi_{1}(X, \zeta)$ is singular, in the form $(m=1,2, \ldots, M)$ on the line $\zeta^{\prime}=\omega_{2} \xi$

$$
\begin{aligned}
& Q_{12}^{(1)}\left(\zeta^{\prime}\right)=-2 \pi \mathrm{i} \sum_{m=1}^{M} q_{12}^{(2 m-1)} \delta\left(\zeta^{\prime}-\zeta_{2 n-1}^{\prime}\right), \\
& Q_{13}^{(1)}\left(\zeta^{\prime}\right)=-2 \pi \mathrm{i} \sum_{m=1}^{M} q_{13}^{(2 m-1)} \delta\left(\zeta^{\prime}-\zeta_{2 n-1}^{\prime}\right) \equiv 0,
\end{aligned}
$$

and on the line $\zeta^{\prime}=-\omega_{3} \xi$

$$
\begin{aligned}
& Q_{12}^{(2)}\left(\zeta^{\prime}\right)=-2 \pi \mathrm{i} \sum_{m=1}^{M} q_{12}^{(2 m)} \delta\left(\zeta^{\prime}-\zeta_{2 n}^{\prime}\right) \equiv 0, \\
& Q_{13}^{(2)}\left(\zeta^{\prime}\right)=-2 \pi \mathrm{i} \sum_{m=1}^{M} q_{13}^{(2 m)} \delta\left(\zeta^{\prime}-\zeta_{2 n}^{\prime}\right) .
\end{aligned}
$$

Now we extend the functional dependence for $Q_{1 j}\left(\zeta^{\prime}\right)$. We focus on the step-function as a possible singularity function

$$
f(x)=\frac{1}{h}(\Theta(x)-\Theta(x-h)),
$$

where $\Theta(x)$ is a Heavyside function. Expanding the Heavyside function $\Theta(x-$ $h$ ) into a Taylor series in the neighborhood of the point $x$

$$
\Theta(x-h)=\Theta(x)+\sum_{n=1}^{\infty}(-1)^{n} \frac{h^{n}}{n !} \Theta^{(n)}(x),
$$

the step-function (4.3) can be rewritten in terms of the derivatives $\delta^{(n)}(x)=$ $\Theta^{(n+1)}(x)$ as follows

$$
\begin{aligned}
f(x) & =\sum_{n=1}^{\infty}(-1)^{n+1} \frac{h^{n-1}}{n !} \Theta^{(n)}(x)=\sum_{n=0}^{\infty}(-1)^{n} \frac{h^{n}}{(n+1) !} \delta^{(n)}(x) \\
& =\delta(x)-\frac{1}{2} h \delta^{(1)}(x)+\ldots .
\end{aligned}
$$

We restrict our consideration to only two terms of the series (4.5) for modelling the singularity functions $Q_{1 j}\left(\zeta^{\prime}\right)$. In the limit $h \rightarrow 0$, the functions $Q_{1 j}\left(\zeta^{\prime}\right)=\frac{-2 \pi \mathrm{i}}{h} q_{1 j}\left(\Theta\left(\zeta^{\prime}\right)-\Theta\left(\zeta^{\prime}-h\right)\right)$ have to be subject to the relations 
(4.1), (4.2). Therefore the singularity functions $Q_{1 j}\left(\zeta^{\prime}\right)$ that we will examine have the following forms $(m=1)$ on the line $\zeta^{\prime}=\omega_{2} \xi$ :

$$
\begin{aligned}
& Q_{12}^{(1)}\left(\zeta^{\prime}\right)=-2 \pi \mathrm{i}\left(q_{12}^{(1)} \delta\left(\zeta^{\prime}-\zeta_{1}^{\prime}\right)-\frac{1}{2} q_{12}^{(1)} h_{1} \delta^{(1)}\left(\zeta^{\prime}-\zeta_{1}^{\prime}\right)\right), \\
& Q_{13}^{(1)}\left(\zeta^{\prime}\right) \equiv 0, \quad \text { i.e. } \quad q_{13}^{(1)} \equiv 0, \\
& h_{1}=h^{(1)}
\end{aligned}
$$

and on the line $\zeta^{\prime}=-\omega_{3} \xi$ :

$$
\begin{aligned}
& Q_{12}^{(2)}\left(\zeta^{\prime}\right) \equiv 0, \quad \text { i.e. } \quad q_{12}^{(2)} \equiv 0, \\
& Q_{13}^{(2)}\left(\zeta^{\prime}\right)=-2 \pi \mathrm{i}\left(q_{13}^{(2)} \delta\left(\zeta^{\prime}-\zeta_{2}^{\prime}\right)-\frac{1}{2} q_{13}^{(2)} h_{2} \delta^{(1)}\left(\zeta^{\prime}-\zeta_{2}^{\prime}\right)\right), \\
& h_{2}=h^{(2)} .
\end{aligned}
$$

Consequently, the spectral data for the continuum spectrum with special singularity functions (4.6), (4.7) are

$$
S=\left\{\zeta_{l}^{\prime}, q_{1 j}^{(l)}, h_{l} ; j=2,3, l=1,2\right\} .
$$

\section{The inverse spectral problem for a special continuum spectrum}

Let us consider the problem of reconstructing the solution $W(X)$ from the spectral data (4.8). This will be straightforward if we can find the vectors $\Phi_{1}(X, T ; \zeta)$. Now we study only the special form of the continuum part of the spectral data $(4.6),(4.7)$, while the variable $\Omega_{d}(X, T ; \zeta)(2.18)$ is considered to be identically zero. For the singularity functions (4.6), (4.7) the relationship (2.17) with (2.19) is reduced to the form (provisionally the time-dependence is not written)

$$
\begin{aligned}
\Phi_{1}(X, \zeta)= & 1-\sum_{l=1}^{2} \sum_{j=2}^{3}\left[q_{1 j}^{(l)} L_{j}\left(X ; \zeta_{l}^{\prime}, \zeta\right) \Phi_{1}\left(X, \omega_{j} \zeta_{l}^{\prime}\right)\right. \\
& \left.+\frac{1}{2} q_{1 j}^{(l)} h_{l}\left(\frac{\partial}{\partial \zeta^{\prime}} L_{j}\left(X ; \zeta^{\prime}, \zeta\right) \Phi_{1}\left(X, \omega_{j} \zeta^{\prime}\right)\right)_{\zeta^{\prime}=\zeta_{l}^{\prime}}\right]
\end{aligned}
$$

where

$$
L_{j}\left(X ; \zeta^{\prime}, \zeta\right) \equiv \frac{\exp \left\{\left[\lambda_{j}\left(\zeta^{\prime}\right)-\lambda_{1}\left(\zeta^{\prime}\right)\right] X\right\}}{\zeta^{\prime}-\zeta} .
$$


We note once again that the relationships (3.14) and (5.1) are similar.

As was proved in Refs.[12-14], the singularities appear in pairs

$$
\zeta_{1}^{\prime}=\omega_{2} \xi_{1}, \quad \zeta_{2}^{\prime}=-\omega_{3} \xi_{1},
$$

where $\xi_{1}$ is a real constant. Moreover

$$
\omega_{2} q_{12}^{(1)}=q_{13}^{(2)} .
$$

It is evident that from (5.3)

$$
h_{1}=\omega_{2} h, \quad h_{2}=-\omega_{3} h,
$$

where $h$ is a real constant.

Here it is convenient to note that the time-evolution of the spectral data appears through (2.16) in the form

$$
\xi_{1}=\text { const }, \quad h=\text { const }, \quad q_{1 j}^{(k)}(T)=q_{1 j}^{(k)}(0) \exp \left(\frac{1}{\mathrm{i} \sqrt{3}} \frac{T}{\xi_{1}}\right) .
$$

The equation (5.1) allows us to define the functions $\Phi_{1}(X, \zeta)$. Indeed, differentiating this equation (5.1) with respect of $\zeta$, and substituting the values $\zeta=\omega_{2} \zeta_{1}^{\prime}, \zeta=\omega_{3} \zeta_{2}^{\prime}$ in the left-hand side of these equations, we obtain a system of four linear algebraic equations in the unknowns $\Phi_{1}\left(X, \omega_{2} \zeta_{1}^{\prime}\right)$, $\Phi_{1}\left(X, \omega_{3} \zeta_{2}^{\prime}\right),\left.\frac{\partial}{\omega_{2} \partial \zeta} \Phi_{1}\left(X, \omega_{2} \zeta\right)\right|_{\zeta=\zeta_{1}^{\prime}},\left.\frac{\partial}{\omega_{3} \partial \zeta} \Phi_{1}\left(X, \omega_{3} \zeta\right)\right|_{\zeta=\zeta_{2}^{\prime}}$. Hence, we could take the function $\Phi_{1}(X, \zeta)$ from Eq. (5.1).

However, there is a more direct method, in which there is no need to obtain the variables $\Phi_{1}\left(X, \omega_{2} \zeta_{1}^{\prime}\right), \Phi_{1}\left(X, \omega_{3} \zeta_{2}^{\prime}\right)$ explicitly. It turns out that we need to calculate only a determinant of some matrix. This approach is similar to the method referred to in $[1,3,10,12-14]$. It is convenient to use new variables introduced by the definition

$$
\Psi_{l}\left(X ; \zeta_{l}^{\prime}\right)=\sum_{j=2}^{3} q_{1 j}^{(l)} \exp \left(\lambda_{j}\left(\zeta_{l}^{\prime}\right) X\right) \Phi_{1}\left(X, \omega_{j} \zeta_{l}^{\prime}\right), \quad l=1,2,
$$

i.e.

$$
\begin{aligned}
& \Psi_{1}\left(X ; \zeta_{1}^{\prime}\right)=q_{12}^{(1)} \exp \left(\lambda_{2}\left(\zeta_{1}^{\prime}\right) X\right) \Phi_{1}\left(X, \omega_{2} \zeta_{1}^{\prime}\right) \\
& \Psi_{2}\left(X ; \zeta_{2}^{\prime}\right)=q_{13}^{(2)} \exp \left(\lambda_{3}\left(\zeta_{2}^{\prime}\right) X\right) \Phi_{1}\left(X, \omega_{3} \zeta_{2}^{\prime}\right)
\end{aligned}
$$


We may rewrite the relationship (5.1) as

$$
\begin{aligned}
\Phi_{1}(X ; \zeta)= & 1-\sum_{l=1}^{2} \frac{\exp \left(-\lambda_{1}\left(\zeta_{l}^{\prime}\right) X\right)}{\zeta_{l}^{\prime}-\zeta} \Psi_{l}\left(X ; \zeta_{l}^{\prime}\right) \\
& +\sum_{l=1}^{2} \frac{1}{2} h_{l} \frac{\partial}{\partial \zeta_{l}^{\prime}}\left(\frac{\exp \left(-\lambda_{1}\left(\zeta_{l}^{\prime}\right) X\right)}{\zeta_{l}^{\prime}-\zeta} \Psi_{l}\left(X ; \zeta_{l}^{\prime}\right)\right) .
\end{aligned}
$$

Here we introduce the notations

$$
\begin{aligned}
L\left(X ; \zeta, \zeta_{l}^{\prime}\right) & \equiv \frac{\exp \left\{\left[\lambda_{1}(\zeta)-\lambda_{1}\left(\zeta_{l}^{\prime}\right)\right] X\right\}}{\zeta_{l}^{\prime}-\zeta} \\
& =-\int_{X} \exp \left\{\left[\lambda_{1}(\zeta)-\lambda_{1}\left(\zeta_{l}^{\prime}\right)\right] X^{\prime}\right\} d X^{\prime},
\end{aligned}
$$

and then

$$
\begin{aligned}
& \frac{\partial}{\partial \zeta_{l}^{\prime}} L\left(X ; \zeta, \zeta_{l}^{\prime}\right)=\int_{X} X^{\prime} \exp \left\{\left[\lambda_{1}(\zeta)-\lambda_{1}\left(\zeta_{l}^{\prime}\right)\right] X^{\prime}\right\} d X^{\prime} \\
& \frac{\partial^{2}}{\partial \zeta \partial \zeta_{l}^{\prime}} L\left(X ; \zeta, \zeta_{l}^{\prime}\right)=\int_{X} X^{\prime 2} \exp \left\{\left[\lambda_{1}(\zeta)-\lambda_{1}\left(\zeta_{l}^{\prime}\right)\right] X^{\prime}\right\} d X^{\prime}
\end{aligned}
$$

Taking into account (2.20), namely

$$
\Phi_{1}(X, \zeta)=1-\frac{1}{3 \lambda_{1}(\zeta)}[W(X)-W(-\infty)]+O\left(\lambda_{1}^{-2}(\zeta)\right)
$$

and (5.6) and (5.7), the following relationship may be found

$$
\begin{aligned}
-\frac{1}{3}[W(X)-W(-\infty)] & =\sum_{l=1}^{2}\left[\exp \left(-\lambda_{1}\left(\zeta_{l}^{\prime}\right) X\right) \Psi_{l}\left(X ; \zeta_{l}^{\prime}\right)\right. \\
& \left.-\frac{1}{2} h_{l} \frac{\partial}{\partial \zeta_{l}^{\prime}} \exp \left(-\lambda_{1}\left(\zeta_{l}^{\prime}\right) X\right) \Psi_{l}\left(X ; \zeta_{l}^{\prime}\right)\right] .
\end{aligned}
$$


Eq. (5.7) with (5.6) in notations (5.8)-(5.10) can be rewritten as follows:

$$
\begin{aligned}
& \exp \left(\lambda_{1}(\zeta) X\right) \Phi_{1}(X ; \zeta)=\exp \left(\lambda_{1}(\zeta) X\right)-\sum_{l=1}^{2} L\left(X ; \zeta, \zeta_{l}^{\prime}\right) \Psi_{l}\left(X ; \zeta_{l}^{\prime}\right) \\
& \quad+\sum_{l=1}^{2} \frac{1}{2} h_{l} \Psi_{l}\left(X ; \zeta_{l}^{\prime}\right) \int_{X} X^{\prime} \exp \left\{\left[\lambda_{1}(\zeta)-\lambda_{1}\left(\zeta_{l}^{\prime}\right)\right] X^{\prime}\right\} d X^{\prime} \\
& \quad+\sum_{l=1}^{2} \frac{1}{2} h_{l} L\left(X ; \zeta, \zeta_{l}^{\prime}\right) \frac{\partial}{\partial \zeta_{l}^{\prime}} \Psi_{l}\left(X ; \zeta_{l}^{\prime}\right) .
\end{aligned}
$$

In contrast to the standard procedure, here it is necessary to take into account the time-evolution for $q_{1 j}^{(k)}(T)$ (5.5). Differentiating Eq. (5.12) with respect to $\zeta$, and substituting the values $\zeta=\omega_{2} \zeta_{1}^{\prime}, \zeta=\omega_{3} \zeta_{2}^{\prime}$ in the left-hand side of these equations, we obtain a system of four linear algebraic equations in the unknowns $\Psi_{l}\left(X ; \zeta_{l}^{\prime}\right), \frac{\partial}{\partial \zeta_{l}^{\prime}} \Psi_{l}\left(X ; \zeta_{l}^{\prime}\right)$ for $l=1,2$. The matrix form of this system of equations is

$$
\mathrm{M} \Psi=\mathrm{b},
$$

where

$$
\Psi=\left(\begin{array}{c}
\Psi_{1}\left(X ; \zeta_{1}^{\prime}\right) \\
\Psi_{2}\left(X ; \zeta_{2}^{\prime}\right) \\
\omega_{3} \frac{\partial}{\partial \zeta_{1}^{\prime}} \Psi_{1}\left(X ; \zeta_{1}^{\prime}\right) \\
\omega_{2} \frac{\partial}{\partial \zeta_{2}^{\prime}} \Psi_{2}\left(X ; \zeta_{2}^{\prime}\right)
\end{array}\right), \quad \mathbf{b}=\left(\begin{array}{c}
q_{12}^{(1)} \exp \left(\omega_{2} \zeta_{1}^{\prime} X\right) \\
q_{13}^{(2)} \exp \left(\omega_{3} \zeta_{2}^{\prime} X\right) \\
q_{12}^{(1)} X \exp \left(\omega_{2} \zeta_{1}^{\prime} X\right) \\
q_{13}^{(2)} X \exp \left(\omega_{3} \zeta_{2}^{\prime} X\right)
\end{array}\right)
$$

The elements of matrix $\mathbf{M}$ are

$$
\begin{aligned}
& M_{11}=1-q_{12}^{(1)} \frac{\exp \left(-\mathrm{i} \sqrt{3} \xi_{1} X\right)}{-\mathrm{i} \sqrt{3} \xi_{1}}-\frac{1}{2} q_{12}^{(1)} h_{1} \int_{X} X^{\prime} \exp \left(-\mathrm{i} \sqrt{3} \xi_{1} X^{\prime}\right) d X^{\prime}, \\
& M_{12}=-q_{12}^{(1)} \frac{\exp \left(2 \omega_{3} \xi_{1} X\right)}{2 \omega_{3} \xi_{1}}-\frac{1}{2} q_{12}^{(1)} h_{2} \int_{X} X^{\prime} \exp \left(2 \omega_{3} \xi_{1} X^{\prime}\right) d X^{\prime} \\
& M_{13}=\frac{1}{2} q_{12}^{(1)} \omega_{2} h_{1} \frac{\exp \left(-\mathrm{i} \sqrt{3} \xi_{1} X\right)}{-\mathrm{i} \sqrt{3} \xi_{1}}
\end{aligned}
$$




$$
\begin{aligned}
M_{14}= & \frac{1}{2} q_{12}^{(1)} \omega_{3} h_{2} \frac{\exp \left(2 \omega_{3} \xi_{1} X\right)}{2 \omega_{3} \xi_{1}}, \\
M_{21}= & -q_{13}^{(2)} \frac{\exp \left(-2 \omega_{2} \xi_{1} X\right)}{-2 \omega_{2} \xi_{1}}-\frac{1}{2} q_{13}^{(2)} h_{1} \int_{X} X^{\prime} \exp \left(-2 \omega_{2} \xi_{1} X^{\prime}\right) d X^{\prime} \\
M_{22}= & 1-q_{13}^{(2)} \frac{\exp \left(-\mathrm{i} \sqrt{3} \xi_{1} X\right)}{-\mathrm{i} \sqrt{3} \xi_{1}}-\frac{1}{2} q_{13}^{(2)} h_{2} \int_{X} X^{\prime} \exp \left(-\mathrm{i} \sqrt{3} \xi_{1} X^{\prime}\right) d X^{\prime} \\
M_{23}= & \frac{1}{2} q_{13}^{(2)} \omega_{2} h_{1} \frac{\exp \left(-2 \omega_{2} \xi_{1} X\right)}{-2 \omega_{2} \xi_{1}}, \\
M_{24}= & \frac{1}{2} q_{13}^{(2)} \omega_{3} h_{2} \frac{\exp \left(-\mathrm{i} \sqrt{3} \xi_{1} X\right)}{-\mathrm{i} \sqrt{3} \xi_{1}}, \\
M_{31}= & -q_{12}^{(1)} \int_{X} X^{\prime} \exp \left(-\mathrm{i} \sqrt{3} \xi_{1} X^{\prime}\right) d X^{\prime} \\
& -\frac{1}{2} q_{12}^{(1)} h_{1} \int_{X} X^{\prime 2} \exp \left(-\mathrm{i} \sqrt{3} \xi_{1} X^{\prime}\right) d X^{\prime}+\frac{T}{\mathrm{i} \sqrt{3} \omega_{3} \xi_{1}^{2}} \\
M_{41}= & \left.-q_{13}^{(2)} \int_{X} q_{13}^{(2)} h_{1} \int_{X} X^{\prime 2} \exp \left(-2 \omega_{2} \xi_{1} X^{\prime}\right) d X^{\prime} \xi_{1} X^{\prime}\right) d X^{\prime} \\
M_{34}= & \frac{1}{2} q_{12}^{(1)} \omega_{3} h_{2} \int_{X} X^{\prime} \exp \left(2 \omega_{3} \xi_{1} X^{\prime}\right) d X^{\prime} \\
& -\frac{1}{2} q_{12}^{(1)} h_{2} \int_{X} X_{12}^{\prime 2} \exp \left(2 \omega_{3} \xi_{1} X^{\prime}\right) d X^{\prime} \\
M_{32}= & -q_{12}^{(1)} \int_{X} X^{\prime} \exp \left(2 \omega_{3} \xi_{1} X^{\prime}\right) d X^{\prime} \\
& X^{\prime} \exp \left(-\mathrm{i} \sqrt{3} \xi_{1} X^{\prime}\right) d X^{\prime} \\
& \\
M_{33} & \\
&
\end{aligned}
$$




$$
\begin{aligned}
M_{42}= & -q_{13}^{(2)} \int_{X} X^{\prime} \exp \left(-\mathrm{i} \sqrt{3} \xi_{1} X^{\prime}\right) d X^{\prime} \\
& -\frac{1}{2} q_{13}^{(2)} h_{2} \int_{X} X^{\prime 2} \exp \left(-\mathrm{i} \sqrt{3} \xi_{1} X^{\prime}\right) d X^{\prime}-\frac{T}{\mathrm{i} \sqrt{3} \omega_{2} \xi_{1}^{2}} \\
M_{43}= & \frac{1}{2} q_{13}^{(2)} \omega_{2} h_{1} \int_{X} X^{\prime} \exp \left(-2 \omega_{2} \xi_{1} X^{\prime}\right) d X^{\prime} \\
M_{44}= & 1+\frac{1}{2} q_{13}^{(2)} \omega_{3} h_{2} \int_{X} X^{\prime} \exp \left(-\mathrm{i} \sqrt{3} \xi_{1} X^{\prime}\right) d X^{\prime}
\end{aligned}
$$

Note that the time-dependence in the matrix elements appears both through $q_{1 j}^{(k)}$ and, in contrast to the standard procedure, through the last terms in $M_{31}$ and $M_{42}$ which appear because $\frac{\partial q_{1 j}^{(k)}}{\partial \xi_{1}} \neq 0$.

Since for any column $j$ of the matrix $\mathbf{M}$ we have

$$
\exp \left(\omega_{k} \xi_{1} X\right) \frac{\partial}{\partial X} M_{i j}=b_{i}, \quad k=\left\{\begin{array}{ll}
2, & \text { if } i=2 n+1 \\
3, & \text { if } i=2 n+2
\end{array},\right.
$$

the sum for (5.11) is

$$
\begin{aligned}
\sum_{l=1}^{2}\left[\exp \left(-\zeta_{l} X\right) \Psi_{l}\left(X ; \zeta_{l}\right)-\frac{1}{2} h_{l} \frac{\partial}{\partial \zeta_{l}} \exp \left(-\zeta_{l} X\right) \Psi_{l}\left(X ; \zeta_{l}\right)\right] \\
=\frac{1}{\operatorname{det} \mathbf{M}} \frac{\partial \operatorname{det} \mathbf{M}}{\partial X} .
\end{aligned}
$$

Finally, from the relation (5.11), the following key relationship may be obtained

$$
W(X)-W(-\infty)=3 \frac{\partial}{\partial X} \ln (\operatorname{det} \mathbf{M}(X)) .
$$

\section{Calculating the determinant of the matrix $M$}

We will prove that the determinant of the matrix $\mathbf{M}$ is given by

$$
\operatorname{det} \mathbf{M}=\left[1+\left(s_{1}+\mathrm{i} r_{1}\left\{X-\frac{T}{3 \xi_{1}^{2}}\right\}\right) \exp \left(\theta_{1}\right)+p_{1} \exp \left(2 \theta_{1}\right)\right]^{2},
$$


where

$$
\begin{aligned}
& s_{1}=c_{1}\left(1+\frac{h}{2 \xi_{1}}\right), \quad r_{1}=\frac{\sqrt{3}}{2} h c_{1}, \quad p_{1}=-\frac{h^{2} c_{1}^{2}}{3 \cdot 2^{4} \xi_{1}^{2}}, \\
& c_{1}=\frac{\beta_{1}}{-\mathrm{i} 2 \sqrt{3} \xi_{1}}, \quad \theta_{1}=-\mathrm{i} \sqrt{3} \xi_{1} X+\frac{T}{\mathrm{i} \sqrt{3} \xi_{1}} .
\end{aligned}
$$

Since the singularities occur in pairs, $\operatorname{det} \mathbf{M}$ is to be a perfect square for some auxiliary function $F$. This statement is not proved directly. However, numerical calculations using the software Maple showed that the matrix $\mathbf{M}$ has two pairs of equal eigenvalues $\lambda_{i}^{(M)}(i=1 \ldots 4)$, i.e. $\lambda_{1}^{(M)}=\lambda_{2}^{(M)}$, $\lambda_{3}^{(M)}=\lambda_{4}^{(M)}$. It is known that the coefficient in $O\left(\lambda^{2}\right)$ in the eigenfunction (eigenpolynomial?) of the $[4 \times 4]$ matrix is written

$$
\sum_{\substack{i, j=1 \\
i<j}}^{4} \operatorname{det}\left(\begin{array}{cc}
M_{i i} & M_{i j} \\
M_{j i} & M_{j j}
\end{array}\right)
$$

On the other hand, under conditions $\lambda_{1}^{(M)}=\lambda_{2}^{(M)}, \lambda_{3}^{(M)}=\lambda_{4}^{(M)}$ this coefficient is equal to $2 \lambda_{1}^{(M)} \lambda_{3}^{(M)}+\left(\lambda_{1}^{(M)}+\lambda_{3}^{(M)}\right)^{2}$. Thus, we have the relationship

$$
\sum_{\substack{i, j=1 \\
i<j}}^{4} \operatorname{det}\left(\begin{array}{cc}
M_{i i} & M_{i j} \\
M_{j i} & M_{j j}
\end{array}\right)=2 \lambda_{1}^{(M)} \lambda_{3}^{(M)}+\left(\lambda_{1}^{(M)}+\lambda_{3}^{(M)}\right)^{2} .
$$

In as much as $\operatorname{Tr} \mathbf{M}=\sum_{i=1}^{4} M_{i i}=2\left(\lambda_{1}^{(M)}+\lambda_{3}^{(M)}\right)$, and $\operatorname{det} \mathbf{M}=\left(\lambda_{1}^{(M)} \lambda_{3}^{(M)}\right)^{2}$, the relationship (6.3) enables us to find the auxiliary function $F=\sqrt{\operatorname{det} \mathbf{M}}$ as follows:

$$
F(X)=\sqrt{\operatorname{det} \mathbf{M}}=\frac{1}{4} \sum_{\substack{i, j=1 \\ i<j}}^{4} M_{i i} M_{j j}-\frac{1}{2} \sum_{\substack{i, j=1 \\ i<j}}^{4} M_{i j} M_{j i}-\frac{1}{8} \sum_{i=1}^{4} M_{i i}^{2} .
$$

Omitting the cumbersome calculation, we finally obtain the relation (6.1).

There are three constants, namely $\xi_{1}, h$ which are are real, and $\beta_{1}$ which could be complex in the general case.

The substitution of the relation (6.4) into (5.16) and the taking into account of the $T$-evolution of the spectral data for the VPE [10] (see also 
(2.16)) allows one to find the solution for the special continuum spectrum (4.6), (4.7) as

$$
W(X, T)-W(-\infty)=6 \frac{\partial}{\partial X} \ln (F(X, T)) .
$$

The problem of selecting the real solution from the complex relation (6.5) is open for study.

\section{The solution for discrete spectral data with two-multiple poles}

The results for the continuum part of the spectral data obtained in Sec. 5 and Sec. 6 can be reduced to the bound state spectrum since the relationships (3.14) and (5.1) are similar to each other. The formal replacements

$$
h \rightarrow \mathrm{i} h, \quad \xi_{1} \rightarrow \mathrm{i} \xi_{1}
$$

lead to the solution (5.16) of the VPE for the discrete spectrum with twomultiple poles (3.14), namely

$$
W(X, T)-W(-\infty)=6 \frac{\partial}{\partial X} \ln (F(X, T))
$$

with auxiliary function

$$
\begin{aligned}
& F(X, T)=1+\left(s_{2}+r_{2}\left\{X+\frac{T}{3 \xi_{1}^{2}}\right\}\right) \exp \left(\theta_{2}\right)+p_{2} \exp \left(2 \theta_{2}\right), \\
& s_{2}=c_{2}\left(1+\frac{h}{2 \xi_{1}}\right), \quad r_{2}=-\frac{\sqrt{3}}{2} h c_{2}, \quad p_{2}=-\frac{h^{2} c_{2}^{2}}{3 \cdot 2^{4} \xi_{1}^{2}}, \\
& c_{2}=\frac{\beta_{1}}{2 \sqrt{3} \xi_{1}}, \quad \theta_{2}=\sqrt{3} \xi_{1} X-\frac{T}{\sqrt{3} \xi_{1}} .
\end{aligned}
$$

The constants $\xi_{1}, h$ are real. There is one arbitrary constant $\beta_{1}$. It is to be real for a real solution.

Note that the auxiliary function $F$ is associated with the $\tau$-function (see, for example, [28-30]).

By taking into account the transformation (7.1), we can apply all mathematical manipulations stated in Sec. 5 and Sec. 6 to the discrete part of the spectral data. 
Since $p_{2}<0$ for arbitrary real $\beta_{1}$, we have $\lim _{X \rightarrow-\infty} F=1$, and $\lim _{X \rightarrow+\infty} F=$ $-\infty$, hence there is $X_{r}$ such that $F\left(X_{r}\right)=0$. Thus the real solution (7.2) with (7.3) is a singular function.

If we determine the value $\beta_{1}$ as an imaginary one, the solutions will be smooth but complex. The selection of the real solutions from complex ones is an open problem.

\section{Conclusion}

Using the VPE as an example, we have shown how, in the IST method, to take into account the two-multiple poles, among single poles, in the discrete part of the spectral data. The special line spectrum of continuum states in the IST method, for which the mathematical procedure is similar to that for the discrete spectrum for two-multiple poles, is considered as well. New solutions are obtained and verified by means of direct substitution into the initial equation by Maple software. The account of the time-dependence is different from the standard procedure.

The important problem which remains is finding the connection between the Lax pairs for the VPE and for the VE, and can be a matter for scientific enquiry in future. This problem is difficult because the solutions of the VPE are single-functions, while the loop-like solutions of the VE can usually be expressed in parametric form only.

\section{References}

[1] Caudrey P J 1982 The inverse problem for a general $N \times N$ spectral equation Physica D 6 51-66

[2] Caudrey P J 1980 The inverse problem for the third order equation $u_{x x x}+q(x) u_{x}+r(x) u=-i \zeta^{3} u$ Phys Lett A $79264-8$

[3] Caudrey P J 1984 Differential and discrete spectral problem and their inverses Wave Phenomena: Modern Theory and Applications eds. C Rogers, T B Moodie (Elsevier Science Publishers, North Holland) 22132

[4] Kaup D J 1980 On the inverse scattering problem for cubic eigenvalue problems of the class $\psi_{x x x}+6 Q \psi_{x}+6 R \psi=\lambda \psi$ Stud Appl Math 62 189-216 
[5] Zakharov V E 1974 On stochastization of one-dimensional chains of nonlinear oscillators Sov Phys JETP 38 108-10

[6] Deift P, Tomei C, Trubowitz E 1982 Inverse scattering and the Boussinesq equation Commun Pure Appl Math 35 567-628

[7] Satsuma J, Kaup D J 1977 A Bäcklund transformation for a higher order Korteweg-de Vries equation J Phys Soc Japan 43 692-7

[8] Hirota R, Satsuma J 1976 A variety of nonlinear network equations generated from the Bäcklund transformation for the Toda lattice Suppl Progr Theor Physics No.59, 64-100

[9] Hirota R 1980 Direct methods in soliton theory Solitons eds. R K Bullough, P J Caudrey (Springer, New York, Berlin) 157-176

[10] Vakhnenko V O, Parkes E J 2002 The calculation of multi-soliton solutions of the Vakhnenko equation by the inverse scattering method Chaos, Solitons and Fractals 13 1819-26

[11] Vakhnenko V O, Parkes E J, 2001 A novel nonlinear evolution equation integrable by the inverse scattering method Rep NAS Ukr No.7, 81-6

[12] Vakhnenko V O, Parkes E J 2012 The singular solutions of a nonlinear evolution equation taking continuous part of the spectral data into account in inverse scattering method Chaos, Solitons and Fractals $\mathbf{4 5}$ $846-52$

[13] Vakhnenko V O, Parkes E J 2012 Solutions Associated with Discrete and Continuous Spectrums in the Inverse Scattering Method for the Vakhnenko-Parkes Equation Prog Theor Phys 127 No.4, 593-613

[14] Vakhnenko V O, Parkes E J 2012 Special singularity function for continuous part of the spectral data in the associated eigenvalue problem for nonlinear equations J Math Phys 53 No.6, 063504(11)

[15] Vakhnenko V A 1992 Solitons in a nonlinear model medium J Phys A: Math Gen 25 4181-7

[16] Parkes E J 1993 The stability of solutions of Vakhnenko's equation $J$ Phys A: Math Gen 26 6469-75 
[17] Vakhnenko V O, Parkes E J 1998 The two loop soliton solution of the Vakhnenko equation Nonlinearity 11 1457-64

[18] Morrison A J, Parkes E J, Vakhnenko V O 1999 The $N$ loop soliton solution of the Vakhnenko equation Nonlinearity 12 1427-37

[19] Ostrovsky L A 1978 Nonlinear Internal Waves in a Rotating Ocean Okeanologia 18 181-91

[20] Hone A N W, Wang J P 2003 Prolongation Algebras and Hamiltonian Operators for Peakon Equations Inverse Problems 19 129-45

[21] Vakhnenko V O, Parkes E J 2004 Periodic and solitary-wave solutions of the Degasperis-Procesi equation Chaos, Solitons and Fractals 201059 73

[22] Musette M, Conte R 1991 Algorithmic method for deriving Lax pairs from the invariant Painlevé analysis of nonlinear partial differential equations J Math Phys 32 1450-7

[23] Clarkson P, Mansfield E L 1995 Symmetry reductions and exact solutions of shallow water wave equations Acta Appi Math 39 245-76

[24] Wazwaz A M $2010 N$-soliton solutions for the Vakhnenko equation and its generalized forms Phys Scr $\mathbf{8 2}$ 065006(7)

[25] Boutet de Monvel A, Shepelsky D 2013 A Riemann-Hilbert approach for the Degasperis-Procesi equation Nonlinearity 262081

[26] Boutet de Monvel A, Shepelsky D 2015 The Ostrovsky-Vakhnenko equation by a Riemann-Hilbert approach J Phys A: Math Theor 48035204

[27] Pontryagin L S, 1962 Ordinary differential equations (Addison-Wesley Publishing Company, London) - 298 p.

[28] Newell A C 1985 Solitons in mathematics and physics (SIAM, Philadelphia) -262 p.

[29] Bao-Feng Feng, Maruno K, Ohta Y 2012 On the $\tau$-functions of the reduced Ostrovsky equation and the $A_{2}^{(2)}$ two-dimensional Toda system J Phys A: Math Theor 45355203 
[30] Bao-Feng Feng, Maruno K, Ohta Y 2013 On the $\tau$-functions of the Degasperis-Procesi equation J Phys A: Math Theor 46045205

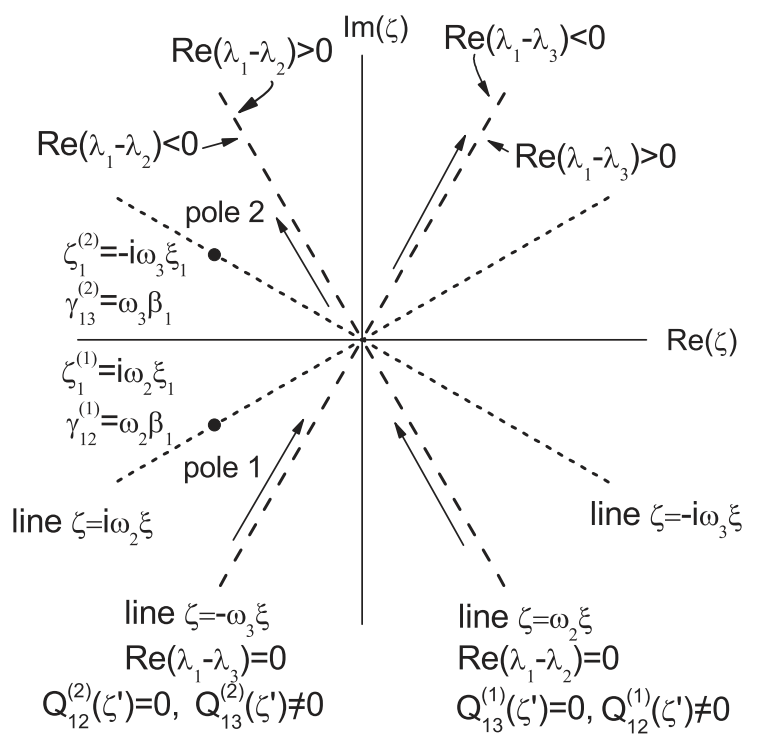

Fig. 1. The regular regions for Jost functions $\phi_{1}(X, \zeta)$ in the complex $\zeta$-plane. The dashed lines determine the boundaries between regular regions. These lines are lines where the singularity functions $Q_{1 j}\left(\zeta^{\prime}\right)$ are given. The dotted lines are the lines where the poles appear. 